American Journal of Nanotechnology 2 (2): 127-131, 2011

ISSN 1949-0216

C 2011 Science Publications

\title{
Nanoscale Pitch Standards Sample Fabricated using Laser-Focused Atomic Deposition
}

\author{
Yan Ma, Tongbao Li, Yajun Zhao, Baowu Zhang, \\ Wen Wu, Yili Xiao, Pingping Zhang and Weigang Gong \\ Department of Physics, \\ Shanghai Key Laboratory of Special Artificial Microstructure Materials and Technology, \\ Tongji University, Shanghai, 20092, China
}

\begin{abstract}
Problem statement: Nanotechnology is already a large sector of industry and science research and it is expected to continue to grow at very fast rate. The determination of absolute measurements of length at the nanometer scale and below is very difficult and expensive. So the nanoscale metrology standard is needed. Approach: The laser-focused atomic deposition is a new way to establish nanoscale pitch standards. When the atoms pass though laser standing wave field, the atoms will change the moving trajectory and be focused to the node (or antinode) of the laser standing wave according to the detuning of laser frequency and atomic resonant frequency. Because of the period of the laser standing wave, laser mask will form the anologue of an array of cylindrical lense. If a substrate is positioned at the focal plane of this lens array, a periodic structure is depositing onto the surface. The period of this structure is $\lambda / 2$ of laser. Results: In this letter, a $425 \mathrm{~nm}$ laser light standing wave is used to focus a beam of chromium atoms to fabricate the nanoscale pitch standards sample of $213 \pm 0.1 \mathrm{~nm}$. The height was $4 \mathrm{~nm}$. The (FWHM) width of $64 \pm 6 \mathrm{~nm}$. Conclusion/Recommendations: The period of this structure is $\lambda / 2$ of laser, whose spatial period can be traced directly to an atomic transtition frequency and the uncertainty possibility is $10^{-5}$, which is fitted to be as the nanopitch standards.
\end{abstract}

Key words: Nanoscale pitch standard, laser-focused atomic deposition, laser cooling, chromium atoms, laser frequency, atomic transtition frequency, focal plane, atomic resonant frequency, atomic transition, periodic structure

\section{INTRODUCTION}

Nanotechnology is already a large sector of industry and science research and it is expected to continue to grow at very fast rate (SAI, 1997). The only difference between nanotechnology and many other fields of science or engineering is that of size. Scientists and engineers want to exploit new physical phenomena that appear when the dimensions of the system are reduced to the nanometre range. Therefore, the precise measurement and control for dimensions of very small objects is the key issue of nanotechnology in which the dimensions of these objects are below $100 \mathrm{~nm}$ and the precision requested frequently is of the order of $0.1 \mathrm{~nm}$. To demonstrate that any product or manufacturing process meets a specified functional demand requires quantitative measurements traceable to an agreed metrology scale. The precision was no longer sufficient; sample-to-sample bias variation is rapidly becoming a significant component of measurement uncertainty. The determination of absolute measurements of length at the nanometer scale and below is very difficult and expensive. In addition, it is difficult to transfer a welldefined macroscopic length standard to the nanometer scale because uncertainties that may be insignificant on the larger scale can become dominant in the transfer process (Postek et al., 1997; Dixon et al., 1999). The technique of laser-focused atomic deposition is a new way to establish nanoscale pitch standards. Artifacts made by this method can be traced directly to an atomic transition frequency. McClelland et al. (2003), have made chromium lines as a highly accurate nanoscale length standard by laser-focused atomic deposition and demonstrated that pitch standards with absolute uncertainties of a few parts in $10^{-5}$ are possible with this technique (McClelland et al., 2003).

In this article we will discuss our experiments which are using neutral chromium atoms to write

Corresponding Author: Tongbao Li, Department of Physics, Shanghai Key Laboratory of Special Artificial Microstructure Materials and Technology, Tongji University, Shanghai, 20092, China 
periodic nanometerscale structures by laser-focused atomic deposition (Timp et al., 1992; McClelland et al., 1993; Sligte et al., 2004; McGowan et al., 1995; Rehse et al., 2000; Fioretti et al., 2005). In these experiments atoms are focused to the desired pattern by the optical dipole force which results from intensity gradients in a light field.

\section{MATERIALS AND METHODS}

Materails: ${ }^{52} \mathrm{Cr}$ is used to deposited on to a silicon wafer $\left(\mathrm{SiO}_{2} / \mathrm{Si}(100)\right)$ with dimensions $15 * 3 \mathrm{~mm} / 0.6$ $\mathrm{mm}$ - thick as the atom optical material. $\mathrm{Cr}$ has good sticking properties on surfaces and the shape of the structure is not expected to level off due to surface diffusion. ${ }^{52} \mathrm{Cr}$ has a transition at $\lambda=425.5 \mathrm{~nm}$ from ${ }^{7} \mathrm{~S}_{3}$ $\rightarrow{ }^{7} \mathrm{P}_{4}{ }^{\circ}$ which can be used for the dipole force manipulation as well as for beam preparation with laser cooling techniques. The transition with a natural line width of $\Gamma=2 \pi * 5 \mathrm{MHz}$ is accessible with a laser system as such: A frequency-doubled CW single-mode Ti: Sapphire laser system, pumped with $10 \mathrm{~W}$ by a LDpumped solid-state laser, typically produced $226 \mathrm{~mW}$ of blue light at $425.5 \mathrm{~nm}$. The laser was locked to the atomic transition $\left({ }^{7} \mathrm{~S}_{3} \rightarrow{ }^{7} \mathrm{P}_{4}{ }^{0}\right)$ using a laser-induced fluorescence technique with stability less than 0.28 $\mathrm{MHz}(<5 \mathrm{MHz})$ (Yan et al., 2006). All experiments of depositing nanopitch standards were carried out in a turbo-molecular pumped vacuum system with typical pressure $10^{-5} \mathrm{~Pa}$. The chromium atomic beam is produced by thermal evaporation out of an orifice $(\Phi$ $=1 \mathrm{~mm}$ ) of a ceramic crucible in a MBE oven at $1650^{\circ} \mathrm{C}$. This leads to a deposition rate of typical $0.0125 \mathrm{~nm} \mathrm{sec}^{-1}$ at a distance of $0.8 \mathrm{~m}$.

The principle of fabracating nanoscale pitch standards: The interaction of near-resonant laser fields with atoms can be shown as (Berggren et al., 1994):

$$
\begin{aligned}
& \mathrm{F}=\frac{\hbar \mathrm{kp}}{1+\mathrm{p}} \Delta \tan (\mathrm{kx})\left[1+\mathrm{v}_{\mathrm{x}} \mathrm{k}\right. \\
& \left.\frac{\Gamma^{2}(1-\mathrm{p})-2 \mathrm{p}^{2}\left(\Delta^{2}+\Gamma^{2} / 4\right)}{\Gamma\left(\Delta^{2}+\Gamma^{2} / 4\right)(1+\mathrm{p})} \tan (\mathrm{kx})\right]
\end{aligned}
$$

Where:

$$
\mathrm{p}=\Gamma^{2} \mathrm{I} / \mathrm{I}_{\mathrm{S}}\left(\Gamma^{2} / 4+\Delta^{2}\right)
$$

Where:
$\Gamma=$ Natural line width of the atomic transition ( $2 \pi * 5 \mathrm{MHz}$ for chromium)

$\Delta=$ Detuning of the laser frequency and atomic transition frequency

$\mathrm{I}=$ Laser intensity

$I_{s}=$ Saturation intensity of the atomic transition (83



$\mathrm{v}_{\mathrm{x}}=$ Atomic velocity of $\mathrm{x}$ direction

$\mathrm{k}=$ Wave vector of laser

The force in Eq. 1 can be think as two part: velocity-dependent and conservative terms. The velocity-dependent terms, named as dissipative force, which arise from Doppler shifts experienced by the atom and from nonadiabatic effects, have been utilized extensively for laser cooling. Another term is called dipole force, which is the interaction of the induced atomic dipole with a gradient in the electric field of laser. The dipole force can be used to focuse atoms. The velocity-dependent terms can be ignored when $\Delta>>\Gamma$ is matching.

The basic principle of the laser-focused atomic to the desired pattern is to use the light-induced force as light mask on free atoms to create nanostructures on a substrate, as Fig. 1 shown (McClelland et al., 1993). When the atoms collimated pass though near-resonant laser standing wave field, the atoms will change the moving trajectory and be focused to the node(or antinode) of the laser standing wave according to the detuning of laser frequency and atomic resonant frequency. Because of the period of the laser standing wave, laser will form the anologue of an array of cylindrical lense. If a substrate is positioned at the focal plane of this lens array, a periodic structure is depositing onto the surface. The period of this structure is $\lambda / 2$ of laser,whose spatial period can be traced directly to an atomic transtition frequency and the uncertainty possibility is $10^{-5}$, which is fitted to be as the nanopitch standard.

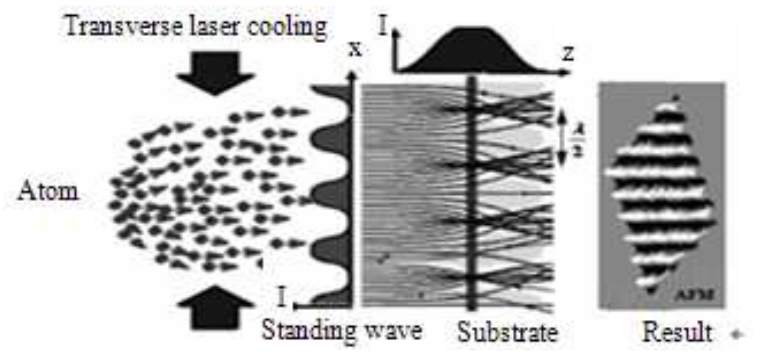

Fig. 1: Schematic of fabracting nanopitch standard 
Am. J. Nanotech., 2 (2): 127-131, 2011

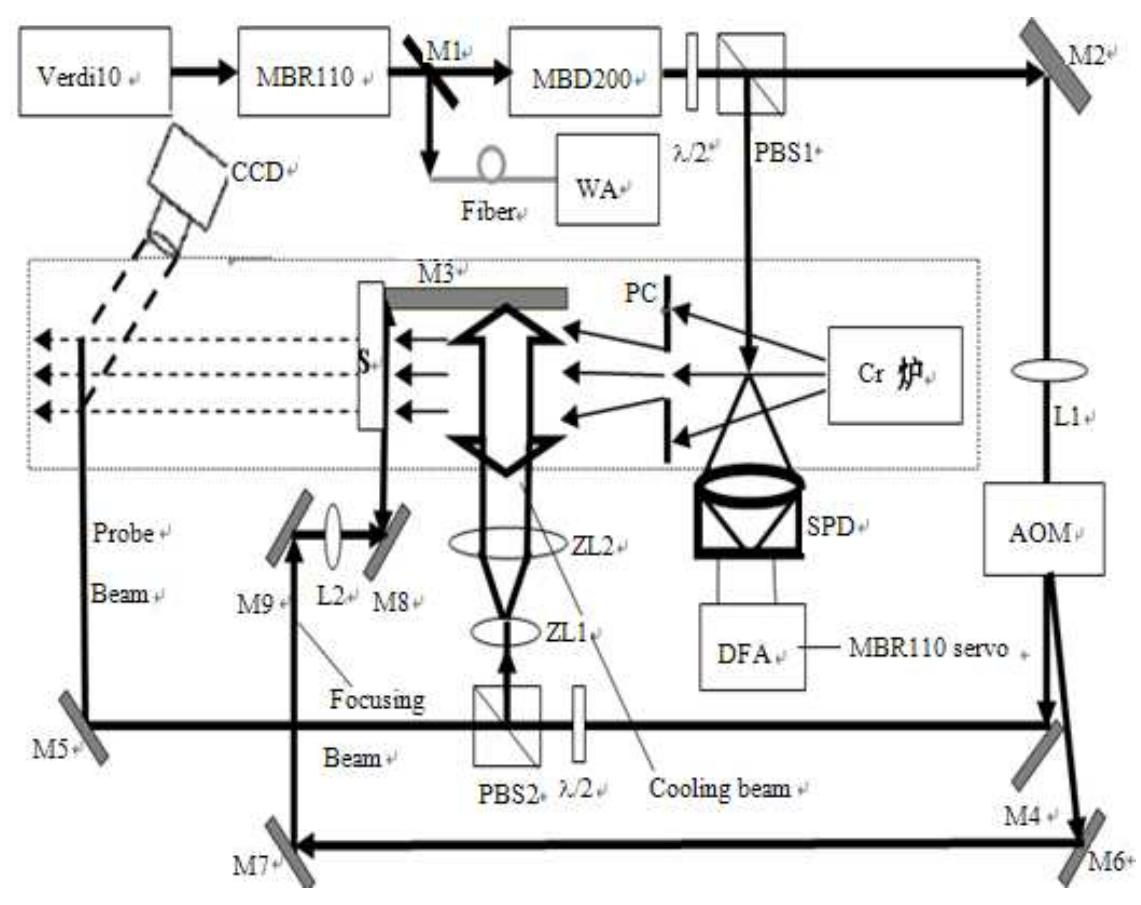

Fig. 2: The set up of fabrication of nanopitch standard

The method of fabricating nanopitch standard: The set up of fabrication of nanopitch standard is shown in Fig. 2. $\mathrm{Cr}^{52}$ is a particularly good atom for the study of atom lithography. In Fig. 2 the frame represents the vacuum chamber. The $\mathrm{Cr}$ beam is produced using a radiatively heated tantalum crucible with a $1 \mathrm{~mm}$ circular aperture. Typically operating temperature is $1600^{\circ} \mathrm{C}$. This beam is mechanically precollimated in the transverse direction to a divergence of about $4.5 \mathrm{mrad}$ with a $1 \mathrm{~mm}$ square aperture $445 \mathrm{~mm}$ from the crucible.

The laser configuration required is within the range of current technology: Chromium has an optical transition from the $\left({ }^{7} \mathrm{~S}_{3}\right)$ ground state to the $\left({ }^{7} \mathrm{P}_{4}^{\circ}\right)$ excited state at a vacuum wavelength of $\lambda=425.5 \mathrm{~nm}$. This transition, with a natural line width of $\Gamma=2 \pi * 5 \mathrm{MHz}$, is accessible with a laser system as such: a $532 \mathrm{~nm}$ LD-pumped intracavity frequencydoubled laser (Coherent Verdi10) with a maximum power output of $10 \mathrm{~W}$ pumps a titanium-sapphire laser (Coherent MBR100) with a maximum output of $1.4 \mathrm{~W}$, tuned at $851 \mathrm{~nm}$. The laser beam at a wavelength of $425.55 \mathrm{~nm}$ is obtained by frequency so-called second harmonic generation of a $851 \mathrm{~nm}$ beam (Coherent MBD200) with a maximum output $220 \mathrm{~mW}$. The 425 $\mathrm{nm}$ beam output from MBD200 is split 3 parts. The first part is used to stabilize the frequency of $425 \mathrm{~nm}$ laser at $- \pm 0.26 \mathrm{MHz}$ away from the $\mathrm{Cr}$ atom resonance by home-made stabilization unit based on the detection of a Laser-Induced Fluorescence (LIF) signal from the chromium beam (Yan et al., 2006). It is because only the red-detuned laser can cool atom and in order to get an optimal performance of the laser cooling. The second part of laser beam is used to collimate $\mathrm{Cr}$ beam transversely. Before it enter into the vacuum chamber, the laser beam is expanded to a $1 / \mathrm{e}^{2}$ width of $24 \mathrm{~mm}$ along the atom beam and a $1 / \mathrm{e}^{2}$ width of $3 \mathrm{~mm}$ transverse to the atom beam using cylindrical lens L1(f $=12.7)$ and L2 (f $=150 \mathrm{~mm})$. This beam profile produces our best atomic beam collimation. Then it enter the vacuum chamber and align itself perpendicular to the atomic beam to better than $1 \mathrm{mrad}$. The collimation beam is retroreflected by a $0^{\circ}$ mirror(reflection $>95 \%$ ) on the other side of the vacuum chamber and aligned parallel to itself to better than $1 \mathrm{mrad}$. Due to the difficulty of perfectly collimating an elliptical beam the retroreflected laser beam expands an additional $5-10 \%$ as it travels the distance to the retroreflecting mirror and back to the interaction region $(\approx 50 \mathrm{~cm})$. For the experimental parameters this additional expansion is inconsequential. The intensity of laser beam is $60 \mathrm{~mW}$. This beam and its retroreflected are used to removing the transverse kinetic energy from the atoms in one dimension, when $\mathrm{Cr}$ atoms pass them.

To obtain a standing light field which is spacially fixed to the substrate surface a laser beam with a beam waist of $100 \mu \mathrm{m}$ is retro-reflected by a $0^{\circ}$ mirror 
(reflection>95\%), which is directly against one face of right-angle prime and perpendicular to the substrate. The optics for the standing wave adjustment as well as the mirror and the substrate are mounted on a mount with five-dimension adjustment so that the standing wave is perpendicular to the atom beam axis. The transverse Gaussian intensity distribution of the standing wave is cut by the substrate in the centre in order to obtain the highest light intensity at the substrate.

To avoid excitation of the atoms in the standing light field, the frequency of the light is shifted with an Acousto-Optic Modulator (AOM) $\Delta=250 \mathrm{MHz}$ to the blue side of the atomic resonance. Then the atoms are focused into the nodes of the standing wave.

\section{RESULTS}

Figure 3 AFM image (Top) of Cr features formed by laser-focused atomic deposition in a standing wave. The image covers a $5 \times 5 \mu \mathrm{m}$ region of the sample. The features are period grating with spacing $212.78 \mathrm{~nm}$ by PSD computation. Line profile is shown by the section analysis (Bottom), the location of being analyzed is indicated on the AFM image using white bright line. Lines are approximately $64 \pm 6 \mathrm{~nm}$ wide and $4 \mathrm{~nm}$ height.

In Fig. 3 an atomic force microscope(AFM Veeco D3100) picture of a chromium nanostructure fabricated with light forces of a standing wave with $\lambda=425.5 \mathrm{~nm}$ is shown. The spacing of the parallel and periodic chromium lines is $213 \pm 0.1 \mathrm{~nm}$, the period is $\lambda / 2$ of the standing wave, which is known with much higher precision than the calibration of AFM. The (FWHM) width of $64 \pm 6 \mathrm{~nm}$ and the height of $4 \mathrm{~nm}$ of the chromium lines are determined by averaging along the lines in this picture, which is not corrected for the shape of the AFM tip. This sample is also measured by NMM (Nanopositioning and Nanomeasuring) in SIMT. The result is shown in Table 1.

The total area with deposited periodic structure is $100 \times 650 \mu \mathrm{m}^{2}$. The area of the chromium structure in the direction of the lines is limited by the laser beam waist to about $100 \mu \mathrm{m}$ and perpendicular to the lines by the breadth of the mechanically-precollimated aperture(center) to $650 \mu \mathrm{m}$. And the structure in the whole area is uniform, which is got from the measurement of every $50 \mu \mathrm{m}$ from the center of the structure. In Fig. 4 a $10 \times 10 \mu \mathrm{m}^{2}$ area is monitored characterizing the uniformity of the periodic structure. The reason we cannot use large area to show the uniformity of the periodic structure is the little measuring range of AFM $(45 \times 45 \mu \mathrm{m})$ and the wide linewidth of grating.

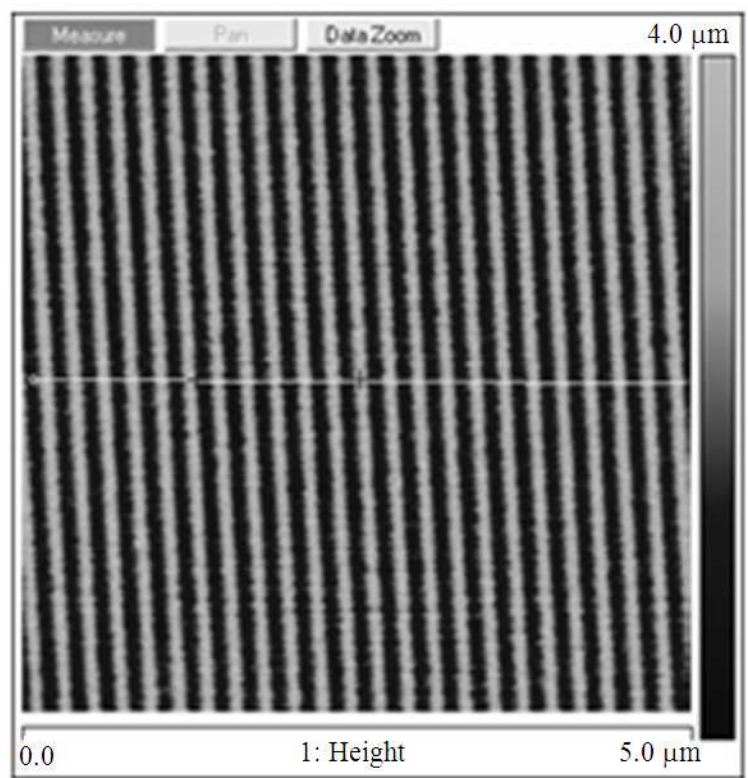

(a)

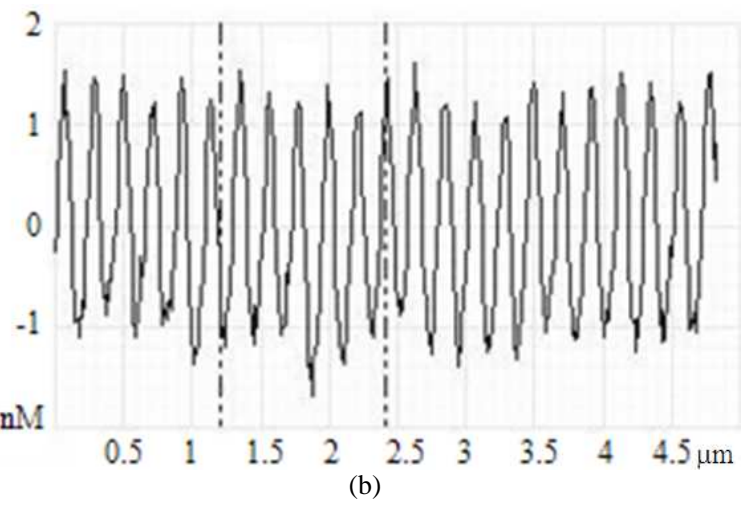

Fig. 3: AFM image (Top) of Cr features formed by laser-focused atomic deposition in a standing wave. The image covers a $5 \times 5 \mu \mathrm{m}$ region of the sample. The features are period grating with spacing $212.78 \mathrm{~nm}$ by PSD computation. Line profile is shown by the Section analysis (Bottom), the location of being analyzed is indicated on the AFM image using white bright line. Lines are approximately $64 \pm 6 \mathrm{~nm}$ wide and $4 \mathrm{~nm}$ height

Table 1: The result is measured by NMM

\begin{tabular}{ll}
\hline Result for mean pitch in $\mathrm{m}:$ & $2.1309 \mathrm{e}-007$ \\
Standard deviation in m: & $4.4651 \mathrm{e}-011$ \\
Mean temperature X axis & $21.1482{ }^{\circ} \mathrm{C}$ \\
Mean temperature Y axis: & $21.0564{ }^{\circ} \mathrm{C}$ \\
Mean temperature Z axis: & $20.4609^{\circ} \mathrm{C}$ \\
Mean air pressure: & $103137.7273 \mathrm{~Pa}$ \\
\hline
\end{tabular}




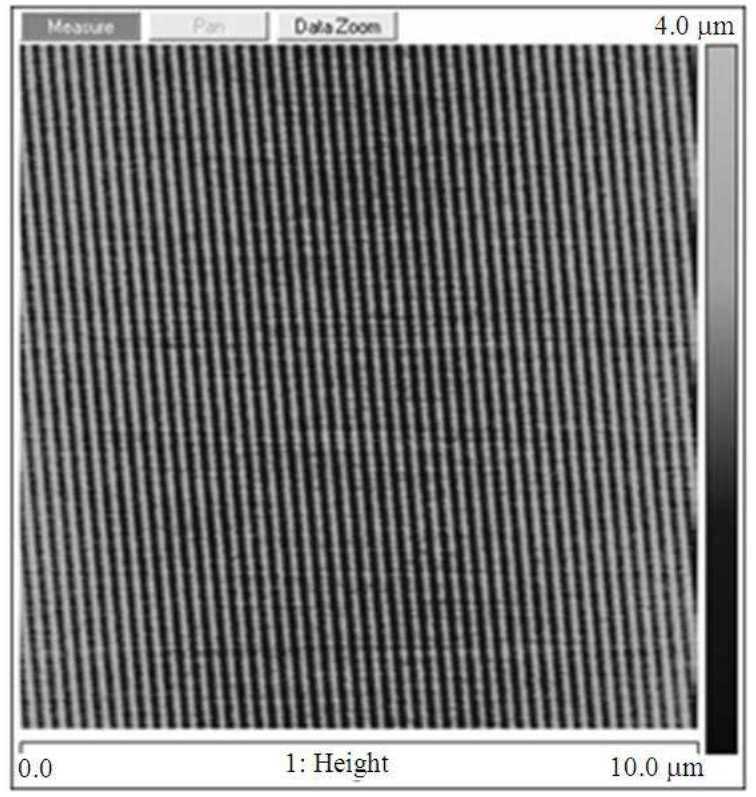

Fig. 4: $10 \times 10 \mu \mathrm{m}$ AFM image of $\mathrm{Cr}$ atomic features showing the uniformity of the periodic structure

\section{DISCUSSION}

For improving the experiment results, the next job will be focused to theoretically analysis the effect of those key parameters on the results.

\section{CONCLUSION}

In conclusion, the technique of laser-focused atomic deposition is used to fabricate nanoscale pitch standard in this article. Chromium atoms are deposited onto a substrate by use of a standing wave. The observed pitch was determined to be $213 \pm 0.1 \mathrm{~nm}$ which coincided with $\lambda / 2$ of the standing wave. The height was $4 \mathrm{~nm}$. The (FWHM) width of $64 \pm 6 \mathrm{~nm}$.

\section{ACKNOWLEDGMENT}

This research Supported by the Shanghai Nanoscience Foundation (0852nm07000,0952nm07000), National natural Science Foundation of China (0804084), National Key Technology $R$ and D Program (2006BAF06B08) and Supported by State Key Laboratory of Precision Measurement Technology and Instruments, Tsinghua University (DL003).

\section{REFERENCES}

Berggren, K.K., M.G. Prentiss, G.L. Timp and R.E. Behringer, 1994. Calculation of atomic positions in nanometer-scale direct-write optical lithography with an optical standing wave. J. Opt. Soc. Am. B., 11: 1166-1176. DOI: 10.1364/JOSAB.11.001166
Dixon, R.G., R. Kining,V.W. Tsai, J. Fu and T.V. Vorburger, 1999. Dimensional metrology witch the nist calibrated atomic force microscope. Proceedings of the SPI, (SPI' 99), Santa Clara, CA., pp: 14-19.

Fioretti, A., A. Camposeo, F. Tantussi, E. Arimondo and S. Gozzini et al., 2005. Atomic lithography with barium atoms. Applied Surf. Sci., 248: 196199. DOI: 10.1016/j.apsusc.2005.03.001

McClelland, J.J., R.E. Scholten, E.C. Palm and R.J. Celotta, 1993. Laser-focused atomic deposition. Science, 262 : 877-880. DOI: 10.1126/science. 262.5135 .877

McClelland, J.J., W.R. Anderson, E. Jurdik, C.C. Bradley and M. Walkiewicz et al., 2003. Accuracy of Nanoscale pitch standards fabricated by laserfocused atomic deposition. J. Res. Natl. Inst. Stand. Technol., 108: 99-113.

McGowan, R.W., D.M. Giltner and S.A. Lee, 1995. Light force cooling, focusing and nanometer-scale deposition of aluminum atoms. Opt. Lett., 20: 2535-2537. DOI: 10.1364/OL.20.002535

Postek, M.T., H. Ho and L. Harrison, 1997. Dimensional metrology at the nanometer level: Combined SEM and PPM. Proceedings of the SPIE, Metrology, Inspection and Process Control for Microlithography, Mar. 10-10, Santa Clara, CA., pp: 250-263. www.nist.gov/manuscript-publicationsearch.cfm?pub_id=820864

Rehse, S.J., R.W. McGowan and S.A. Lee, 2000. Optical manipulation of Group III atoms. Applied Phys. B: Lasers Opt., 70: 657-660. DOI: 10.1007/s003400050876

SAI, 1997. The National Technology Roadmap for Semiconductors. Semiconductor Industry Association,

Sligte, E.T., B. Smeets, K.M.R.V.D. Stam, R.W. Herfst and P.V. Straten et al., 2004. Atom lithography of Fe. Applied Phys. Lett., 85: 4493-4495.

Timp, G., R.E. Behringer, D.M. Tennant, J.E. Cunningham and M. Prentiss, 1992. Using light as a lens for submicron, neutral-atom lithography. Phys. Rev. Lett., 69: 1636-1639. PMID: 10046275

Yan, M., Z. Bao-Wu, Z. Chun-Lan, M. Shan-Shan and L. Fo-Sheng et al., 2006. Experimental study of laser collimation of $\mathrm{Cr}$ beam. Acta Phys. Sin., 55: 4086-4090. 